\title{
Vehicle-Mounted Photovoltaic System Energy Management in Intelligent Transportation Systems: A Maximum Power Point Tracking Control
}

\author{
Jingao Wang, 1 Qifei Liu $\mathbb{D}^{\mathrm{D}},{ }^{1}$ and Silan Jing ${ }^{2}$ \\ ${ }^{1}$ College of Information Science and Engineering, Northeastern University, Shenyang, Liaoning 110819, China \\ ${ }^{2}$ College of Business Administration, Northeastern University, Shenyang, Liaoning 110819, China \\ Correspondence should be addressed to Qifei Liu; lqf184848458@163.com
}

Received 22 January 2021; Revised 26 May 2021; Accepted 24 July 2021; Published 5 August 2021

Academic Editor: Dan Selisteanu

Copyright (c) 2021 Jingao Wang et al. This is an open access article distributed under the Creative Commons Attribution License, which permits unrestricted use, distribution, and reproduction in any medium, provided the original work is properly cited.

\begin{abstract}
Electric vehicles have become the main contributor in terms of reducing fuel consumption and $\mathrm{CO}_{2}$ emission. Although the government is vigorously promoting the use of electric vehicles worldwide, the range anxiety still impedes the rapid development of electric vehicles, especially when air-conditioning also adds battery power consumption and aggravates the range anxiety. To this end, this paper proposes an improved vehicle-mounted photovoltaic system energy management in intelligent transportation systems, which is a maximum power point tracking control system. Meanwhile, since the power of solar panels is usually relatively small and the power changes at any time, low power density and poor controllability are difficult to avoid. In order to solve this problem, this paper offers a tracking control method to improve the output efficiency of solar panels. For improving photovoltaic conversion efficiency and maximizing output power, traditional photovoltaic power panels are often dominated by a centralized maximum power point tracing control, which is named MPPT. Although the cost under this case is lower, the output power of all photovoltaic panels cannot be maximized under the condition of uneven illumination or local mismatch. To improve the situation, a micro-scale inverter is proposed to provide MPPT control of photovoltaic modules, which can effectively improve the output power of each photovoltaic panel. Moreover, our MPPT algorithm is applicable to cloud shadow, building shadow, and shade, and it is more suitable for the car roof. Firstly, the Diode 5-parameter model is used to deduce the $I-U$ equation of the photovoltaic module considering shadow shading, and then the real-time 5 parameter equation is formed by using the measured data group and selected. The reasonable initial value is used to iteratively solve the real-time value of 5 parameters, which is further to judge the masking situation. The maximum power point (MPP) is solved directly by the mathematical method based on the mathematical model of $I-U$ relation mathematics, and the DC-DC circuit is used to adjust the running point to MPP. Unlike the traditional MPPT method, the method in this paper is based on the physical model of solar cells, and MPP tracking is based on mathematical methods. Based on this, it does not need to cause multiple interference to the circuit, and the tracking efficiency is high. Finally, the relative experimental results are provided to verify the performance of the proposed method.
\end{abstract}

\section{Introduction}

Because of the high temperature in a vehicle in summer, the vehicle owner is very uncomfortable when entering it. In particular, when children are locked in a car, they are frequently killed by heat. Based on this problem, using a photovoltaic cell to drive the air-conditioner and cool the car is very effective. The cooperative control of photovoltaic modules such as solar cells is the key to achieve it because photovoltaic panels can get extra energy and direct power to the air-conditioner without using the electricity in the dynamic battery, which can reduce mileage anxiety. However, the photovoltaic panels provide very limited power due to the limitation of the car roof area. Thus, it is significant to find the maximum power point of onboard photovoltaic panels to increase its power generation and apply this method to the solar energy controller in Figure 1. In this section, the most important difficulty to realize cooperative 




Figure 1: PV-based vehicle temperature control system.

control is how to track the maximum power point of photovoltaic panels.

According to the external environment, the output power of solar cell varies with the operating point of voltage and current, but the output power has a maximum value, and at this time, the photoelectric conversion efficiency reaches the peak [1]. Therefore, photovoltaic development should be specialized in maximum power point tracking control to raise the efficiency in solar power stations [2]. Since the solar cell is a nonlinear system, the relationship between the output current and voltage is a complex implicit nonlinear system [3], so it is difficult to use mathematical model tracking (MPPT), the maximum rate of photovoltaic directly [4].

The general MPPT method does not need to consider the intelligent disturbance optimization control of the model, such as the disturbance observation method increasing conductance method $[5,6]$. The advantage is they do not consider the complexity of the model, and logic is simple [7], but the disadvantage is that it will cause multiple disturbances of the circuit, the control speed is slow, and the adjustment cannot be realized in one step [8]. The improved disturbance observation method can improve the tracking speed by a reasonable adaptive step size control strategy, such as changing the disturbance step size according to the slope of the $P$ - $U$ curve, and can alleviate the oscillation of the circuit at the maximum power point [9].

The traditional centralized inverter utilizes a master MPPT controller to control the total output power of the photovoltaic power station by disturbance observation [10]. At this point, the current constraint between the series solar panels in the photovoltaic array and the voltage constraint between the parallel groups will lead to this condition. While the total output power reaches the top, the corresponding output power of each solar panel does not [11] at its respective maximum power points, especially when the illumination and cloud shadow obscured, or other mismatch occurs [12]. Although some modern algorithms work very well in tracking the maximum power points [13-15], these methods can only be used for static photovoltaic films, and the equipment cost is high. And also, they are suitable for large-scale photovoltaic power generation but are not used for vehicles. As a consequence, a sort of inverter for controlling a series of photovoltaic panels and a microinverter for controlling solar cell veneer are proposed in this paper. Both deeply enhance the utilization rate of illumination; the former cost is lower, but the latter has higher reliability and photoelectric conversion rate. Expansion is easier too [16].

Aiming at the vehicle microinverter installed in the solar cell veneer, this paper considered its mechanism mathematical model and quantified the process of finding the maximum power point. The maximum power point of three solar panels is tracked by the mathematical method by using the measured data. This avoids the traditional MPPT method to the circuit caused by multiple disturbances, which is more suitable for improving the power generation efficiency of vehicle photovoltaic panels with less power.

\section{Five-Parameter Model for Photovoltaic Modules}

2.1. Five-Parameter Model for Photovoltaic Modules. Figure 2 shows the single diode equivalent circuit of the solar cell. It can be seen that the solar cell is equivalent to a current source parallel to a diode considering the series parallel loss. In Figure 2, $I, I_{p h}, I_{D}$, and $I_{s h}$ stand for the output current, the photogenerated current, the equivalent diode current, and the current flowing over the equivalent parallel resistor, respectively. $R_{s h}, R_{s}$ are equal to parallel resistance and series resistance; $U_{D}, U$ are equal to diode voltage and output voltage, respectively.

The equivalent circuit corresponds to five parameter model expressions: 


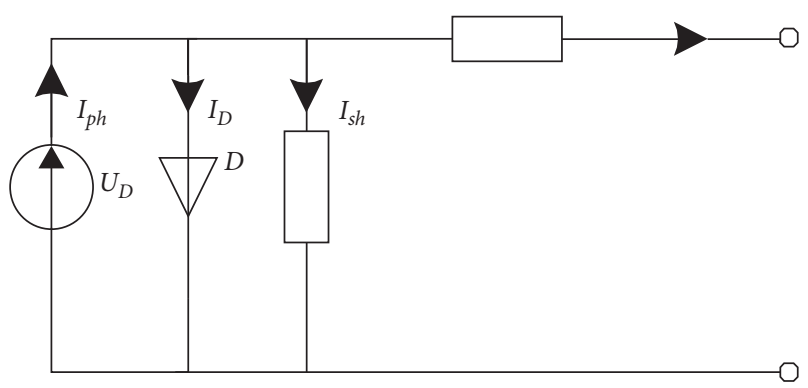

FIgURE 2: Equivalent circuit of the photovoltaic cell.

$$
\begin{aligned}
& I=I_{p h}-I_{s}\left[e^{\left(q\left(U+I R_{S}\right) / A k T\right)}-1\right]-\frac{U+I R_{S}}{R_{s h}}, \\
& I=I_{p h}-I_{s}\left[e^{q\left(U+I R_{S}\right) / A k T}-1\right]-\frac{U+I R_{S}}{R_{s h}} .
\end{aligned}
$$

In this form, $q$ is the electrical power constant, whose value is $1.602 \times 10^{-19} \mathrm{C}$; $k$ is the Boltzmann constant, whose value is $1.381 \times 10^{-23} \mathrm{~J} / \mathrm{K} ; A$ is the diode characteristic fitting coefficient, whose value is from 1 to 2 ; and $I_{s}$ is the equivalent diode saturated current.

Formula (1) can be regarded as an equation with five undetermined parameters, which are $I_{p h}, R_{s h}, a, I_{s}$, and $R_{s}(a$ represents comprehensive fitting coefficient, equal to $q / A k T$ and $T$ represents real-time temperatures)

2.2. Relation of Parameters to Irradiance and Temperature. The correction at different temperatures and irradiance form is

$$
\begin{aligned}
I_{p h} & =\left(\frac{S}{S_{\text {ref }}}\right)\left[I_{p h, \text { ref }}+C_{T}\left(T-T_{\text {ref }}\right)\right], \\
I_{s} & =I_{s, \text { ref }}\left(\frac{T}{T_{\text {ref }}}\right) e^{\left[\left(q E_{g} / k A\right)\left(\left(1 / T_{\text {ref }}\right)-(1 / T)\right)\right],} \\
R_{S} & =R_{s, \text { ref }}\left(\frac{T}{T_{\text {ref }}}\right)^{3}\left[1-\beta \ln \left(\frac{S}{S_{\text {ref }}}\right)\right] \\
R_{s h} & =R_{s h, \text { ref }}\left(\frac{S}{S_{\text {ref }}}\right) .
\end{aligned}
$$

In this form, $I_{p h, r e}$ is photogenerated current under standard operating conditions; $S$ equals real-time irradiance; $S_{\text {ref }}$ is irradiance under standard conditions ; $C_{T}$ is temperature coefficient, available from the manufacturer; $I_{\text {ref }}$ is diode saturation current in standard condition; $T_{\text {ref }}$ is temperature under standard conditions; and $E_{g}$ is the bandgap, depending on the solar cell material. In this paper, the bandgap width of silicon is $1.12 \mathrm{eV} ; \beta$ is the correction factor; and $\beta$ is 0.217 .

The variation of these five parameters with temperature and irradiance is investigated, and the parameter values under standard conditions (ring temperature $25^{\circ} \mathrm{C}$, illumination $1000 \mathrm{~W} / \mathrm{m}^{2}$ ) are compared and calculated:
(1) Temperature is 0.99 times of standard temperature and irradiance

(2) Irradiance is the standard value and the temperature is 1.01 times the standard condition (the temperature change is about $3 \mathrm{~K}$, considering the temperature difference within $1 \mathrm{~min}$ in extreme cases)

(3) The temperature is the standard temperature, the irradiance is the parameter value of 0.7 times under the standard condition (the irradiance variation is $300 \mathrm{~W} / \mathrm{m}^{2}$, considering the amplitude difference within $1 \mathrm{~h}$ under normal conditions)

(4) The irradiance is the standard value and the temperature is 1.03 times the parameter value under the standard condition (the temperature change is about $9 \mathrm{~K}$, considering the temperature difference within $1 \mathrm{~h}$ in extreme cases)

From Table 1, it can be seen that when the time scale is measured in minutes, the variation of each parameter with irradiance and temperature can be ignored. If the time scale is counted in hours, the change rate of each parameter is much smaller than that of irradiance, so the effect of temperature can be ignored within $1 \mathrm{~h}$. Meanwhile, in the 5 parameters including $a, I_{s}$, and $R_{s}$, the irradiance has little effect, and it can be considered as a definite value; it means that only $I_{p h}$ and $R_{s h}$ changed within $1 \mathrm{~h}$. Two calculation modes can be divided according to the time scale. If considering the whole point of timing (such as 10:00 11:00), the time length between every two whole points can be specified as a large time scale; then, the large time scale is divided into 60 parts. That is $1 \mathrm{~h}$ per minute.

Within the same large time scale, each small time scale corresponds to different $I_{p h}, R_{s h}$. At this time, only the $I_{p h}, R_{s h}$ parameters should be identified to simplify the calculation and improve the calculation speed. The whole point needs to consider the large time scale and $a, I_{s}$, and $R_{s}$ change accordingly. At this time, we need to calculate 5 parameters.

2.3. Collaborative 5-Parameter Model of $N$ Photovoltaic Modules. The photovoltaic module containing $N$ monomers is demonstrated in Figure 3(a). Each monomer is divided into $M$ groups, each of which is connected with a bypass diode. An equivalent circuit of a series unit on the component board is shown in Figure 3(b).

Considering the masking situation, when one or several monomers in a set are obscured, the current will go through directly through the bypass diode. The terminal voltage of each group is the forward conduction voltage of the diode, and the silicon tube is $0.3 \mathrm{~V}$, so the conduction voltage $U_{D D}=0.3 \mathrm{~V}$.

Taking into account $N=60, M=20$ groups of panels, a solar panel has $N_{1}$ cells without shelter, and $N_{2}$ cells with shelter; the equivalent diode voltage of each group of cells without shelter is shown in the following formula: 
TABLE 1: Relationship between the parameters and conditions.

\begin{tabular}{|c|c|c|c|c|c|c|}
\hline $\begin{array}{l}\text { Number of different environmental } \\
\text { conditions }\end{array}$ & $\begin{array}{c}\text { Temperature irradiance } \\
\text { conditions }\end{array}$ & $I_{p h}(\mathrm{~A})$ & $I_{\mathrm{s}}(\mathrm{mA})$ & $a$ & $R_{\mathrm{s}}(\mathrm{mW})$ & $R_{\mathrm{sh}}(\mathrm{W})$ \\
\hline Standard situation & $\begin{array}{l}S=S_{\text {ref }} \\
T=T_{\text {ref }}\end{array}$ & $I_{p h, \mathrm{ref}}$ & $I_{s, \text { ref }}$ & $a$ & $R_{s, \text { ref }}$ & $R_{s h, \text { ref }}$ \\
\hline 1 & $S=0.99 S_{\text {ref }}, T=T_{\text {ref }}$ & $0.99 I_{p h, \text { ref }}$ & $I_{s, \text { ref }}$ & $a$ & $1.002 R_{s, \text { ref }}$ & $0.99 R_{s h, \mathrm{ref}}$ \\
\hline 2 & $S=S_{\text {ref }}, T=1.01 T_{\text {ref }}$ & $I_{p h, \mathrm{ref}}+0.01 C_{T}$ & $1.03 I_{s, \text { ref }}$ & $0.99 a$ & $1.01 R_{s, \mathrm{ref}}$ & $R_{s h, \mathrm{ref}}$ \\
\hline 3 & $S=0.7 S_{\text {ref }}, T=T_{\text {ref }}$ & $0.7 I_{p h, \mathrm{ref}}$ & $I_{s, \text { ref }}$ & $a$ & $1.08 R_{s, \text { ref }}$ & $0.7 R_{s h, \mathrm{ref}}$ \\
\hline 4 & $S=S_{\text {ref }}, T=1.03 T_{\text {ref }}$ & $I_{p h, \mathrm{ref}}+0.03 C_{T}$ & $1.09 I_{s, \text { ref }}$ & $0.97 a$ & $1.03 R_{s, \text { ref }}$ & $R_{s h, \text { ref }}$ \\
\hline
\end{tabular}



(a)

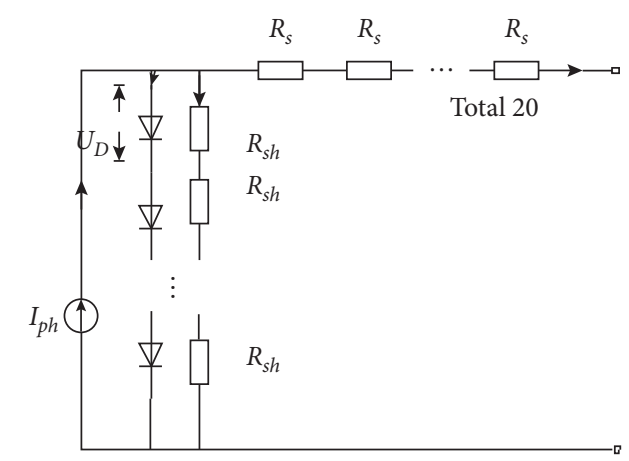

(b)

FIGURE 3: Schematic diagram of PV panel and equivalent circuit of a unit in PV pane. (a) Single board diagram. (b) Schematic diagram of the equivalent circuit of a series unit of solar cell veneer and component board.

$$
U_{D}=\frac{U-\left(N_{2} / 20\right) U_{D D}+I N_{1} R_{S}}{N_{1}}=\frac{U}{N_{1}} \frac{N_{2} U_{D D}}{20 N_{1}}+I R_{S}
$$

Considering the $U-I$ relation in the case of shelter,

$$
I=I_{p h}-I_{S}\left[e^{a\left(\left(U / N_{1}\right)-\left(N_{2} U_{D D} / 20 N_{1}\right)+I R_{S}\right)}-1\right]\left(\frac{U}{N_{1}}-\frac{N_{2} U_{D D}}{20 N_{1}}+I R_{S}\right) \frac{1}{R_{s h}} .
$$
$N_{2}$ :

There are four different combinations for panels $N_{1}$ and

(1) $N_{1}=0, N_{2}=60, N_{1} / N_{2}=0$

(2) $N_{1}=20, N_{2}=40, N_{1} / N_{2}=12$

(3) $N_{1}=40, N_{2}=20, N_{1} / N_{2}=2$

(4) $N_{1}=60, N_{2}=0, N_{1} / N_{2}=\infty$

When the battery chip is covered, if the $U$ is normal, $N_{1}=60, N_{2}=0$; if the value of the $U$ changes to about a third, $N_{1}=40, N_{2}=20$; if the $U$ value changes to about $2 / 3$ of the original, $N_{1}=20, N_{2}=40$; and if the $U$ is very small, the panels do not work, considering the masking condition MPPT is not required. A five-parameter $I-U$ relation can be obtained.

\section{Analysis of the Characteristics of $P-U$ Curves considering Shadows}

3.1. Solar Cell P-U and I-U Analysis. P-U and $I-U$ curves are determined under certain illumination and temperature conditions, as shown in Figure 4. PMP represents the power at the maximum power point, IMP represents the current at the maximum power point, and $U_{\mathrm{MP}}$ and $U_{\mathrm{OC}}$ represent the voltage at the maximum power point and the open-circuit voltage, respectively. There are 3 special points on the curve in Figure 4. Output short-circuit point corresponds to when the output voltage $U$ is 0 ; output open-circuit point corresponds to when the output current is 0 ; and maximum power point corresponds to output power maximum point.

The power expression is shown in the following formula:

$$
P=U I
$$

The derivative of the $P$ to the $U$ at the maximum power point is 0 :

$$
\frac{\mathrm{d} P}{\mathrm{~d} U}=0 .
$$

At this point, the output power is the maximum output power: 




Figure 4: $I-U$ curve and $P-U$ curve of PV array.

$$
P_{\mathrm{MP}}=U_{\mathrm{MP}} I_{\mathrm{MP}}
$$

The photoelectric conversion rate and the utilization rate of light energy are the highest. When the initial operating point is $\left(U_{1}, I_{1}\right)$ and the corresponding power is $P_{1}$, it can be seen from the $P-U$ curve that the operating point can be adjusted to the maximum power point ( $\left.U_{\mathrm{MP}}, I_{\mathrm{MP}}\right)$ through the external control device.

\subsection{Bimodal Characteristics of Component Plate under Partial} Shadow. When the solar panel is partially shaded, the $P-U$ curve presents a bimodal characteristic, as shown in Figure 5 , in which $U_{\text {JMP }}$ represents the voltage at the local maximum power point and $P_{\mathrm{JMP}}$ represents the power at the local maximum power point. Conventional perturbation optimization $M_{\mathrm{PPT}}$ methods have the probability of tracing to the local peak points, which are the pseudo-maximum points.

\section{Acquisition of Maximum Power Points}

4.1. Parameter Identification. Aiming at the fault of parameter identification by parameter correction method, a parameter identification method which uses real-time gaugement data is proposed in this paper. According to the analysis in Section 3.2, the parameter identification is divided into two cases to simplify the computation:

(1) Adjust the external circuit of the photovoltaic module five times and then measure voltage and current values of 5 groups; thus, determine the corresponding 5-parameter model according to the voltage situation;

(2) According to the 5-parameter model and the 5 sets of voltage and current values, the 5 equations are determined as shown in formula (8). We take this approach to solve the 5 parameters and get the $U-I$ relationship at the whole point. Iterative calculations only take advantage of the previous result at the whole point and then use it as the initial value

$$
I_{i}=I_{p h}-I_{S}\left[e^{a\left(\left(U / N_{1}\right)-\left(N_{2} U_{D D} / 20 N_{1}\right)+I R_{S}\right)}-1\right]-\left(\frac{U_{i}}{N_{1}}-\frac{N_{2} U_{D D}}{20 N_{1}}+I_{i} R_{S}\right) \frac{1}{R_{s h}}, \quad i=1,2,3,4,5
$$

In this formation, $I_{i}$ and $I_{s}$ are the current values in the $i$ set data, $\mathrm{A}$ is the saturation current of the equivalent diode; and $U_{i}$ is the voltage value in group $i$ data.

For any moment, assuming that the irradiance is the same as the previous integral moment, we replace 3 parameters of the five which are only affected by temperature change with integral values. By measuring two sets of voltage and current values, we established two equations for the two parameters greatly affected by irradiance. The expression of the two parameters is shown in formula (9), and we found the $U-I$ relation at any time is obtained:

$$
\left\{\begin{aligned}
I_{\mathrm{ph}} & =\frac{\left[I_{1}+I_{s}\left(e^{V_{1}-1}\right)\right]\left(U_{2}-\left(N_{2} U_{D D} / 20\right)+N_{1} I_{2} R_{S}\right)}{\left(U_{2}+N_{1} I_{2} R_{S}\right)-\left(U_{1}+N_{1} I_{1} R_{S}\right)}-\frac{\left[I_{2}+I_{S}\left(e^{V_{2}}-1\right)\right]\left(U_{1}-\left(N_{2} U_{D D} / 20\right)+N_{1} I_{1} R_{S}\right)}{\left(U_{2}+N_{1} I_{2} R_{S}\right)-\left(U_{1}+N_{1} I_{1} R_{S}\right)} \\
\frac{1}{R_{\mathrm{sh}}} & =\frac{N_{1}\left(I_{1}-I_{2}\right)+N_{1} I_{S}\left(e^{V_{1}}-e^{V_{2}}\right)}{\left(U_{2}+N_{1} I_{2} R_{S}\right)-\left(U_{1}+N_{1} I_{1} R_{S}\right)}
\end{aligned}\right.
$$

In this formula, 


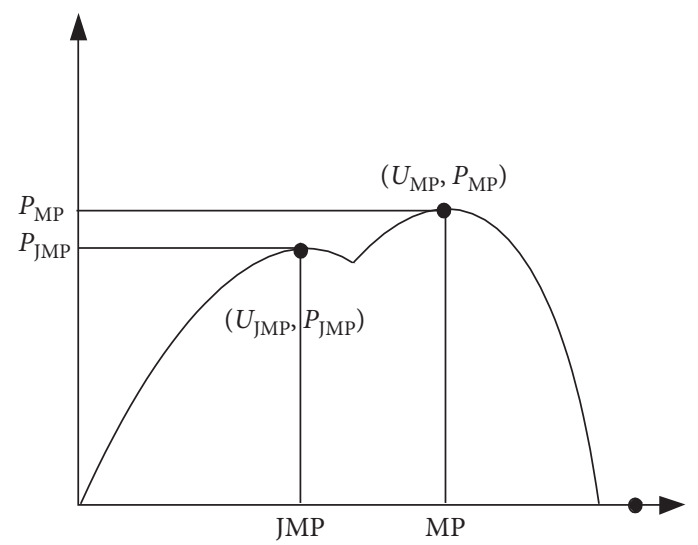

Figure 5: Bimodal characteristics of $P$ - $U$ curves of PV modules under shadow shading.

$$
\begin{aligned}
& V_{1}=a\left(\frac{U_{1}}{N}-\frac{N_{1} U_{D D}}{20 N_{1}}+I_{1} R_{S}\right), \\
& V_{2}=a\left(\frac{U_{2}}{N}-\frac{N_{1} U_{D D}}{20 N_{1}}+I_{2} R_{S}\right) .
\end{aligned}
$$

4.2. Maximum Power Point Positioning. Simplify the I-U curve formulas (4) to (11):

$$
\ln \left[\frac{\left(I_{p h}-I\right) N_{1} R_{s h}-\left(U-\left(N_{2} U_{D D} / 20\right)+I N_{1} R_{s}\right)}{N_{1} R_{s h} I_{s}}+1\right]=a\left(\frac{U}{N_{1}}-\frac{N_{2} U_{D D}}{20 N_{1}}+I R_{s}\right) .
$$

Formulas (5) and (11) derive $U$ on both sides separately and further simplify the following available formula:

$$
\left[-\frac{U}{N_{1}}+I\left(R_{s}+R_{s h}\right)\right] \frac{1}{a}=\left(I_{p h}-I\right) R_{s h}\left(\frac{U}{N_{1}}-I R_{s}\right)-\left(\frac{U}{N_{1}}-\frac{N_{2} U_{D D}}{20 N_{1}}+I R_{s}+R_{s h} I_{s}\right)\left(\frac{U}{N_{1}}-I R_{s}\right) .
$$

Combine formulas (12) and (11), and the maximum power point $\left(U_{\mathrm{MP}}, I_{\mathrm{MP}}\right)$ is solved out.

If assuming that

$$
\frac{U}{N_{1}}-\frac{N_{2} U_{D D}}{20 N_{1}}+I R_{s}=x, \quad I=y,
$$

then

$$
\begin{aligned}
\frac{U}{N_{1}}-I R_{s} & =x+2 y R_{s}+\frac{N_{2} U_{D D}}{20 N_{1}}, \quad I=y, \\
U & =\left(X+\frac{N_{2} U_{D D}}{20 N_{1}}-I R_{s}\right) N_{1} .
\end{aligned}
$$

Formulas (11) and (12) can be reduced to the following formulas:

$$
\begin{gathered}
-\frac{1}{a}\left[x+y\left(2 R_{s}+R_{s h}\right)+\frac{N_{2} U_{D D}}{20 N_{1}}\right]=\left[\left(I_{p h}-y\right) R_{s h}-x+R_{s h} I_{s}\right]\left(x+2 y R_{s}+\frac{N_{2} U_{D D}}{20 N_{1}}\right), \\
x(K+1)=\frac{A k T}{q} \ln \left\{\frac{\left[I_{p h}-y(K)\right] R_{s h}-x(K)}{R_{s h} I_{s}}+1\right\} .
\end{gathered}
$$

In these formulas, $x$ and $y$ are the replacement variables during the solution and $K$ is the iteration number.
Formula (15) can be regarded as a quadratic equation about $y$, and it has 2 solutions generally, which proved the 


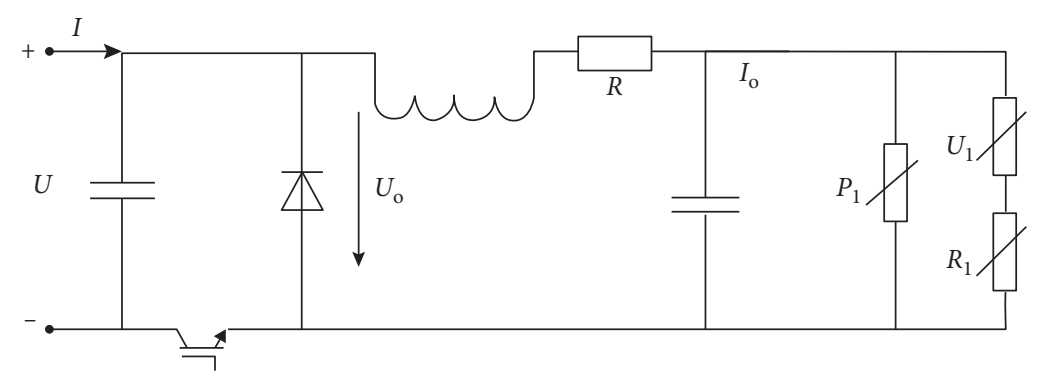

FiguRE 6: External buck circuit and load equivalent circuit diagram.

TABLe 2: Data of current and voltage of PV panel.

\begin{tabular}{lccccc}
\hline$N_{2} / N_{1}$ & $\left(U_{1}, I_{1}\right)$ & $\left(U_{2}, I_{2}\right)$ & $\left(U_{3}, I_{3}\right)$ & $\left(U_{4}, I_{4}\right)$ & $\left(U_{5}, I_{5}\right)$ \\
\hline 0 & $17.6,5.3$ & $23.0,5.2$ & $27.5,5.0$ & $29.1,4.3$ & $30.85,3.6$ \\
0.5 & $11.4,5.3$ & $14.5,5.2$ & $17.9,4.9$ & $19.7,4.2$ & $21.0,3.3$ \\
2 & $5.9,5.2$ & $7.11,5.2$ & $8.33,5.0$ & $9.22,4.3$ & $9.86,4.0$ \\
\hline
\end{tabular}

bimodal characteristic mentioned in Section 3.2. It means that there are 2 points when the derivation of power to voltage is 0 . Comparing the power value of the corresponding solution, we can abandon the "pseudo-maximum point."

Taking the maximum power point of a moment as the initial iteration value and putting $x(K), y(K)$ to (16), we can find the corresponding $x\left(K_{1}\right)$ and then bring it back and find out $y\left(K_{1}\right)$. Under the condition that $U$ and $I$ are greater than 0 , if $y\left(K_{1}\right)$ has two values, then compare the corresponding power values and take a set of values with the large corresponding power. Iterating it to the error within the allowable range, the corresponding $I-U$ is the $I-U$ at the maximum power.

4.3. Tracing the Maximum Power Point. Assuming that the load is a combination of constant power, invariant voltage, and invariant resistance, it is connected to the photovoltaic module by Buck circuit as shown in Figure 6.

The voltage and current at the end of the system in relation to the duty cycle $\alpha$ can be obtained from the equal power characteristic before and after the Buck circuit, as shown in the following formula:

$$
\alpha=\frac{U_{1}+\sqrt{U_{1}^{2}+4\left(U I+P_{1}\right) R_{1}}}{2 U} .
$$

After calculating the corresponding $I-U$ value of the maximum power point, the corresponding duty cycle is procured, and the adjustment of the duty cycle can jump to the working point at the vicinity of the maximum power point directly.

\section{Simulation Verification}

Data in this article is simulated by PVsyst6.61. We establish a system that adopts a microinverter structure in PVsyst6.61 and each photovoltaic panel is equipped with a microinverter. Each PV module consists of 60 batteries.
We simulated a module board in PVsyst6.61 which has 6 series battery cells, and every two parallels have bypass diodes.

Select a panel in PVsyst to verify the feasibility of this method under the condition of no shading, 2 shadings, and 4 shadings, respectively. The simulation of cloud shadow masking and the internal mismatch is carried out through artificial masking, which causes some interference to the circuit. The data of panel current and voltage ( 3 groups in total) is collected as shown in Table 2.

By using the data in Table 2 and the quasi-Newton iteration method, the initial values of photovoltaic 5 parameters are $\left[I_{p h}, I_{s}, a, R_{s}, R_{s h}\right]=[6,0.000001,25,0.0002$, $6000]$. The initial values are gained from the factory parameters of photovoltaic modules, which is the corresponding 5 parameter values under the standard working condition. When the quasi-Newton method is used for iterative calculation, the selection of the initial values is related to the iterative speed and the convergence of the results. In the actual operation, the result of the last parameter identification can be taken as the initial value of the next iteration to ensure the reliability of the iteration and obtain the parameters. The identification results are shown in Table 3.

The photovoltaic characteristic curve equation can be written by using the 5 parameters. According to the steps described in Section 4.2, the maximum power points can be calculated by mathematical equations as shown in the MPP1 in Table 4. However, when the interference exists for a long time, the maximum power point tracked by the inverter actually can be measured as shown in the MPP2 in Table 4. Meanwhile, we also experimented this method under the condition of sunny days, cloudy days, and cloud shading days; the detailed data are in Table 5.

The value of the maximum power point calculated by the method described in this paper is consistent with the value of the maximum power point tracked by the MPPT control strategy basically. However, because there are errors during measure and model, the results will not be completely consistent. 
TABle 3: Result of parameter identification.

\begin{tabular}{lccccc}
\hline Experimental group & $I_{p h}(\mathrm{~A})$ & $I_{s}(\mathrm{~mA})$ & $a$ & $R_{s}(\mathrm{~mW})$ & 3.3 \\
No shelter & 5.4 & 2.2 & 2.1 & 25.5 & 4.2 \\
2-line shelter & 5.3 & 2.5 & 25.7 & 3.8 & 8891 \\
4-line shelter & 5.2 & & 9192 \\
\hline
\end{tabular}

TABLE 4: Maximum power point computation.

\begin{tabular}{lccccc}
\hline Experimental group & $I_{p h}(\mathrm{~A})$ & $U(\mathrm{~V})$ & $I(\mathrm{~A})$ & $P(\mathrm{~W})$ & Relative power error (\%) \\
\hline \multirow{2}{*}{ No shelter } & MPP1 & 28.2 & 4.8 & 135.36 & -2.04 \\
\hline \multirow{2}{*}{ 2-line shelter } & MPP2 & 29.4 & 4.7 & 138.18 & 89.18 \\
\multirow{2}{*}{ 4-line shelter } & MPP1 & 18.2 & 4.9 & 83.04 & 7.39 \\
& MPP2 & 17.3 & 4.8 & 43.68 & -2.1 \\
\hline
\end{tabular}

TABle 5: Maximum power point computation under three different real-weather environments.

\begin{tabular}{lccc}
\hline Experimental group & $U(\mathrm{~V})$ & $I(\mathrm{~A})$ & $P(\mathrm{~W})$ \\
\hline Sunny days & 27.92 & 4.65 & 129.83 \\
Cloudy days & 9.12 & 4.70 & 42.86 \\
Cloud shading days & 16.56 & 4.77 & 78.99 \\
\hline
\end{tabular}

\section{Conclusion}

Using the five-parameter model of the solar cell to model the mechanism of photovoltaic module and the mathematical model to solve the maximum power point directly, the conclusion is as follows:

Different combinations of temperature and irradiance correspond to different values of 5 parameters; the disturbance circuit can gain equations for solving 5 parameters by measuring the values of voltage and current. In order to get the real-time value of 5 parameters, the maximum power point is solved directly by a mathematical equation. Compared with the traditional disturbance observation method, this direct mathematical method has fewer interference times with the circuit and it can provide faster MPPT speed.

The selection of initial values is related to the iteration speed and convergence of the results. The identification results of the parameters at the last moment offer the best iterative initial value for the next moment, and the iterative results have preferable convergence.

By dividing the unused time scale and utilizing the relationship between 5 parameters and temperature and irradiance, the parameters with less influence can be regarded as invariant for a period of time, so only two parameters need to be identified. Different time scales correspond to different parameter identification numbers; thus, this method simplifies the calculation process.

\section{Data Availability}

The data used to support the findings of this study are included within the article.

\section{Conflicts of Interest}

The authors declare that there are no conflicts of interest regarding the publication of this paper.

\section{Acknowledgments}

The project was supported by the Key Program of National Natural Science Foundation of China (61433004).

\section{References}

[1] M. A. Ghasemi, H. M. Foroushani, and F. Blaabjerg, "Marginal power-based maximum power point tracking control of photovoltaic system under partially shaded condition," IEEE Transactions on Power Electronics, vol. 35, no. 6, 2020.

[2] K. Liu and T. Z. Ji, X. Y. Li, X. F. He, and M. Zhang, Study of maximum power point tracking control based on GRNN neural network," Advanced Materials Research, vol. 989-994, pp. 3427-3432, 2014.

[3] F. Xu, R. Zhang, L. Wu, and H. Xu, "Self- adaption BP natural network for the maximum- power-point tracking in photovoltaic," Acta Energiae Solaris sinica, vol. 33, no. 3, pp. 468$472,2012$.

[4] J. Y. Shi, D. Y. Zhang, L. T. Ling et al., "Dual-algorithm maximum power point tracking control method for photovoltaic systems based on grey wolf optimization and goldensection optimization," Journal of Power Electronics, vol. 18, no. 3, pp. 841-852, 2018.

[5] S. K. Kollimalla and M. K. Mishra, "Variable perturbation size adaptive P\&O MPPT algorithm for sudden changes in irradiance," IEEE Transactions on Sustainable Energy, vol. 5, no. 3, pp. 718-728, 2014.

[6] S. Tang, Y. Sun, and Y. Chen, "An enhanced MPPT method combining fractional-order and fuzzy logic control," IEEE Journal of Photovoltaics, vol. 7, no. 2, 2017.

[7] S. VeerapenaH. Wena et al., "A novel global maximum power point tracking algorithm for photovoltaic system with variable perturbation frequency and zero oscillation," Solar Energy, vol. 181, pp. 345-356, 2019.

[8] S. A. Ansari and J. S. Moghani, "Soft switching flyback inverter for photovoltaic AC module applications," IET Power Electronics, vol. 13, p. 83, 2019. 
[9] S. Twaha, J. Zhu, L. Maraaba, K. Huang, B. Li, and Y. Yan, "Maximum power point tracking control of a thermoelectric generation system using the extremum seeking control method," Energy, vol. 10, no. 12, 2017.

[10] J. M. Kwon, K. H. Nam, and K. H. Nam, "High-efficiency module-integrated photovoltaic power conditioning system," IET Power Electronics, vol. 2, no. 4, pp. 410-420, 2009.

[11] A. Ymeri and S. Mujović, "Optimal location and sizing of photovoltaic systems in order to reduce power losses and voltage drops in the distribution grid," International Review of Electrical Engineering, vol. 12, no. 6, pp. 498-504, 2017.

[12] M. Luthfansyah, S. Suyanto, and A. B. M. Bangura, "Evaluation and comparison of DC-DC power converter variations in solar panel systems using maximum power point tracking (MPPT) flower pollination algorithm (FPA) Control," in Proceedings of the 1st International Conference on Renewable Energy Research and Challenge, p. 606, Banyuwangi, Indonesia, 2020.

[13] M. Eltamaly Ali, "A novel musical chairs algorithm applied for MPPT of PV systems," Renewable and Sustainable Energy Reviews, vol. 3, p. 146, 2021.

[14] Q. Liu, S. Pang, and Y. Gao, "An intelligent dual-mode control strategy for extending MPPT operation interval of DFIG," Electric Power Components and Systems, vol. 48, no. 16-17, 2021.

[15] Ngo Van-Quang-Binh, L. Mohsen, A. Rabeh, J. Houssem, K. Ohshima, and K. Mehrdad, "Improved krill herd algorithm based sliding mode MPPT controller for variable step size $\mathrm{P} \& \mathrm{O}$ method in PV system under simultaneous change of irradiance and temperature," Journal of the Franklin Institute, vol. 358, no. 7, pp. 3491-3511, 2021

[16] S. Kouro, J. I. Leon, D. Vinnikov et al., "Grid connected photovoltaic systems: an overview of recent research and emerging PV converter technology," IEEE Industrial Electronics Magazine, vol. 9, no. 1, pp. 47-61, 2015. 